\title{
Tracking single baculovirus retrograde transportation in host cell via quantum dot-labeling of virus internal component
} CrossMark

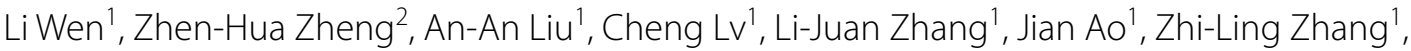 \\ Han-Zhong Wang ${ }^{2}$, Yi Lin ${ }^{1 *}$ and Dai-Wen Pang ${ }^{1,2}$
}

\begin{abstract}
Background: Quantum dot (QD)-based single virus tracking has become a powerful tool for dissecting virus infection mechanism. However, only virus behaviors at the early stage of retrograde trafficking have been dynamically tracked so far. Monitoring of comprehensive virus retrograde transportation remains a challenge.

Results: Based on the superior fluorescence properties of QDs and their labeling of virus internal component, the dynamic interactions between baculoviruses and all key transportation-related cellular structures, including vesicles, acidic endosomes, actins, nuclear pores and nuclei, were visualized at the single-virus level. Detailed scenarios and dynamic information were provided for these critical interaction processes.

Conclusions: A comprehensive model of baculovirus retrograde trafficking involving virus endocytosis, fusion with acidic endosome, translocation to nuclear periphery, internalization into nucleus, and arriving at the destination in nucleus was proposed. Thus the whole retrograde transportation of baculovirus in live host cells was elucidated at the single-virus level for the first time.
\end{abstract}

Keywords: Quantum dots, Single virus tracking, Baculovirus, Retrograde transportation, Host cells

\section{Background}

Dissection of virus-cell interactions is of great significance for the prevention of virus-related diseases [1, 2]. As the prerequisite for infection, the movements of viruses from cell surface to nucleus, the so-called retrograde transportation, has aroused extensive attention in past decades [3-5]. Recently, owing to the superior brightness and stability of quantum dots (QDs) [6], QDbased single virus tracking (SVT) has become a powerful tool for investigating infection dynamics of viruses at the single-virus level by providing in situ and real-time evidences [7-11]. To date, by employing SVT based on

\footnotetext{
*Correspondence: ylin@whu.edu.cn

${ }^{1}$ Key Laboratory of Analytical Chemistry for Biology and Medicine

(Ministry of Education), College of Chemistry and Molecular Sciences,

State Key Laboratory of Virology, The Institute for Advanced Studies,

and Wuhan Institute of Biotechnology, Wuhan University, Wuhan 430072,

People's Republic of China

Full list of author information is available at the end of the article
}

QD-labeling of virus external envelope, information on the early stages of virus retrograde transportation has been provided [6-9]. However, since virus envelope would dissociate during virus fusion with acidic endosome, only the behaviors of viruses before the fusion have been dynamically tracked so far. The visualization of comprehensive virus retrograde trafficking, consisting of infection events both before and after the fusion, remains a challenge.

SVT via QD-labeling of virus internal component can be one perfect option. We have proposed a mild approach for preparing recombinant baculovirus (RBV) with QDs labeled capsid (QDs-RBV) [11]. By infecting host Spodoptera frugiperda 9 (Sf9) cells with recombinant bacmids, RBV with biotinylated capsid was obtained and subsequently labeled with streptavidin conjugated QDs. Herein, based on the superior properties of QDs [6], baculovirus retrograde transportation in host Sf9 cells was monitored at the single-virus level. Moreover, via 
QD-labeling of virus internal component, combined with drug inhibition assays, individual QDs-RBV interacting with all the suspectable retrograde transportation-related cellular structures [12] was dynamically tracked in real time. Thus detailed elucidation of the whole retrograde journey of single baculovirus infection was enabled.

\section{Methods}

\section{Cell culture and QDs-RBV preparation}

Sf9 cells were cultured at $28{ }^{\circ} \mathrm{C}$ in Grace's medium supplemented with $10 \%(\mathrm{v} / \mathrm{v})$ fetal bovine serum (Gibco). QDs-RBVs were prepared as in our previous report [11]. Briefly, RBVs with internal biotinylated capsids were produced by infecting Sf9 cells with recombinant bacmids. RBV stock was amplified by infecting Sf9 cells with RBVs at a multiplicity of infection (MOI) of 1 . Then RBVs were incubated with $2 \mathrm{nM}$ of streptavidin conjugated $\mathrm{CdSe}_{x} \mathrm{Te}_{1-x}$ QDs (SA-QDs, Wuhan Jiayuan Quantum Dots Co., Ltd.) for $0.5 \mathrm{~h}$ at $4{ }^{\circ} \mathrm{C}$. QDs-RBVs were purified with sucrose density gradient ultracentrifugation. Afterwards, QDs-RBVs were diluted in PBS and filtered through a $0.45 \mu \mathrm{m}$ film (Millipore). Wild type baculoviruses (WBVs) operated in the same procedures served as control.

\section{Transmission electron microscopy (TEM) imaging}

Fifteen microliter virus solutions were laid on carboncoated copper grids for $10 \mathrm{~min}$ adsorption. The copper grids were placed in a freeze-dryer to remove all the water. Hitachi H-7000 FA transmission electron microscope was used for examining the viruses at $200 \mathrm{kV}$.

\section{Colocalization assays}

Sf9 cells cultured overnight were incubated with QDsRBVs at a MOI $=5$ for $10 \mathrm{~min}$ at $4{ }^{\circ} \mathrm{C}$, followed by the fixation with $4 \%$ paraformaldehyde for $25 \mathrm{~min}$ at room temperature. Anti-VP39 antibody (1:2000, Biosciences) or anti-GP64 antibody (1:2000, Biosciences) was added to the cells for $1 \mathrm{~h}$ incubation at $37{ }^{\circ} \mathrm{C}$. The cells were then washed with PBS and incubated with dylight 649-conjugated secondary antibody (Thermo) for $45 \mathrm{~min}$ at $37^{\circ} \mathrm{C}$. After PBS washing as above, the cells were used for colocalization analysis. WBV operated in the same procedure served as the controls.

\section{Fluorescence labeling of cellular structures}

Sf9 cells cultured overnight were treated with $5 \mu \mathrm{g} /$ $\mathrm{mL}$ CellMask, $5 \mu \mathrm{g} / \mathrm{mL}$ LysoTracker Green or $5 \mu \mathrm{g} /$ $\mathrm{mL}$ Hoechst 33342 (Invitrogen) to label the cytomembranes, acidic endosomes or nuclei, respectively. For the labeling of actins or nuclear pore complexes (NPC), Sf9 cells were incubated with $4 \%$ paraformaldehyde and $0.1 \%$ Triton X-100, followed by the addition of $5 \mu \mathrm{g} / \mathrm{mL}$
Phalloidin-FITC (Invitrogen) to label actins or anti-NPC antibody (Covance) and dylight 649-conjugated secondary antibody (Abbkine) to label NPC.

\section{Cytotoxicity assays}

Sf9 cells were inoculated into a 96-well for a stationary culture. Fresh medium containing 1, 2.5, 5, 10, $20 \mu \mathrm{g} /$ $\mathrm{mL}$ of Hoechst 33342 were added for 90 min incubation, respectively. For cytotoxicity assays of Hoechst 33342 at the working concentration $(5 \mu \mathrm{g} / \mathrm{mL})$, fresh medium containing $5 \mu \mathrm{g} / \mathrm{mL}$ of Hoechst 33342 were added for 10, 20, 40, 60, 90, 120 min incubation, respectively. Fresh medium containing no Hoechst 33342 served as control. Then MTT [3-(4,5-dimethylthiazol-2-yl)-2,5-diphenyltetrazolium bromide] solution $(0.5 \mathrm{mg} / \mathrm{mL})$ was added for $4 \mathrm{~h}$ incubation. The solutions were changed into 120 $\mu \mathrm{L}$ of DMSO for $5 \mathrm{~min}$ incubation. OD value was measured at the wavelength of $570 \mathrm{~nm}$. Cell viability was calculated by this formula: $\left(\mathrm{OD}_{\text {treated }} / \mathrm{OD}_{\text {control }}\right) \times 100 \%$.

\section{Drug inhibition assays and microinjection}

Hundred nanomolar Bafilomycin A1, $10 \mu \mathrm{g} / \mathrm{mL}$ Cytochalasin D or $10 \mu \mathrm{M}$ Nocodazole (Sigma) were used in drug inhibition assays. The supernatants extracted from the drug treated cells at $60 \mathrm{~h}$ postinfection were collected for the determination of virus titers with $50 \%$ tissue culture infective dose (TCID50) assays [13]. Thus the yields of the propagated baculovirus were measured. An Eppendorf FemtoJet injection system (Eppendorf AG) was used for microinjecting $0.5 \mathrm{mg} / \mathrm{mL}$ FITC labeled wheat germ agglutinin (FITC-WGA) (AMSBIO LLC).

\section{Fluorescence imaging and data analysis}

Fluorescence signals were detected under the $100 \times$ objective of a spinning-disk confocal microscope (Andor Revolution XD) equipped with an EMCCD (Andor iXon DV885 K). Hoechst 33342, 605 nm SA-QDs and Dylight 649 were excited with 405, 561 and $640 \mathrm{~nm}$ laser and detected using 447/60, 605/20 and 685/40 nm filter, respectively. CellMask/LysoTracker Green/FITC were excited with $488 \mathrm{~nm}$ and detected using 525/50 nm filter. For real-time tracking, the images were recorded with a frame interval of $2 \mathrm{~s}$, an exposure time of $500 \mathrm{~ms}$ and a readout time of $17.8 \mathrm{~ms}$. The interactions of viruses with vesicles, acidic endosomes, actins and nuclei were imaged at 0, 12, 25 and $45 \mathrm{~min}$ after virus adding and cell staining, respectively. A stage here means that viruses were interacting with one kind of cellular structures such as vesicles, acidic endosomes, actins and nuclei. For each stage, at least three parallel tracking experiments were conducted, each of which was carried out by preparing a confocal dish containing cells and viruses for imaging with the same manipulation. Typically, 30 individual 
trajectories (length $>15 \mathrm{~min}$ ) from these dishes were analyzed for each stage.

Line profiles [4] (indicating the dependence of dualchannel signals distributed on the circle) were analyzed with Imaging-Pro-Plus (IPP). Image J was used for calculating Manders coefficients $\mathrm{tMr}$ and $\mathrm{tMg}$, and intensity correlation quotient (ICQ) [4] values from at least 800 viruses. ICQ value ranging from 0.1 to 0.5 indicates a strong covariance of the dual-channel signals.

For the calculation of fit parameters, the trajectories of QDs-RBVs were analyzed with IPP to obtain positions and velocities [14]. Time averaged mean square displacement (MSD) curves that correspond to single trajectories [15] were calculated by the user-written program with Matlab based on virus positions [16]. The obtained MSD ( $y$-axis) and the corresponding time ( $x$-axis) were subsequently imported to Origin for fitting based on the equations $\mathrm{MSD}=4 D \tau+(V \tau)^{2}+$ constant (indicating a directed movement) or MSD $=4 D \tau^{\alpha}+$ constant (indicating an anomalous diffusion movement). $D$ and $V$ are the diffusion coefficient and the fitting velocity respectively. $\tau$ represents time. $\alpha$ is an exponent $(\alpha<1)$ [17]. For each fit parameter, values obtained from 30 individual trajectories were used for the calculation of mean \pm S.D. with Origin.

\section{Results and discussion}

\section{Characterization of QDs-RBV}

QDs-RBV (Fig. 1a) was prepared as in our previous report [11] (see also the "Methods"). QDs-RBV can be easily obtained by incubating RBV with SA-QDs, probably because the internal capsid would be partially exposed with the loss of some loose envelope during virus purification with ultracentrifugation $[18,19]$. Moreover, the infectivity of viruses was maintained during the labeling and subsequent purification processes [11]. As shown in Fig. 1b, no obvious difference was observed in the emission spectra of QDs-RBVs and QDs, indicating that the labeling of RBVs did not affect the fluorescence properties of QDs. TEM images showed that for QDsRBVs, QDs appeared around RBVs (Fig. 1c). On the contrary, no QD was found with WBVs (Fig. 1d), suggesting that QDs were attached only to RBVs. To evaluate the labeling efficiency and virus integrity after the labeling, QDs-RBVs were added to Sf9 cells for attachment and subsequently immunolabeled on baculovirus capsid protein VP39 or envelope protein GP64. As shown in Fig. 1e, $\mathrm{h}, \mathrm{QD}$ signals colocalized with the immunofluorescence of both VP39 and GP64. In contrast, QD signals were not observed in the control. The corresponding line profiles [4] showed that the dual-channel signals from QDs-RBVs closely related to each other (Fig. 1f, i), further proving the binding of QDs to RBVs. The colocalization also implied that QDs-RBV maintained both its envelope and capsid. According to the quantitative analysis [4] (see also the Methods), a high QD-labeling efficiency of $c a$. 93\% was obtained (Fig. 1g, j).

\section{Dynamic interaction between QDs-RBVs and vesicles}

SVT was employed to explore QDs-RBV retrograde transportation in host cells. Firstly, Sf9 cells were infected with QDs-RBVs and labeled with CellMask (a cytomembrane dye). As shown in Fig. 2a, signals of QDs-RBVs were circled by those of cytomembrane, indicating that QDs-RBVs could be effectively internalized into Sf9 cells. Based on immunological [20] and inhibition assays [21], baculovirus would undergo cell uptake through endocytosis. By analyzing and segmenting 30 valid trajectories of virus internalization [22, 23], it was found that single QDs-RBV typically experienced three internalization steps (Fig. 2b): being trapped into a vesicle formed from cytomembrane for $60 \pm 6 \mathrm{~s}$ (step 1 ), moving towards cell interior for $40 \pm 4 \mathrm{~s}$ (step 2) and fusing with another intracellular vesicle containing virus for $50 \mathrm{~s} \pm 6 \mathrm{~s}$ (step 3). According to MSD vs time curves and velocity vs time curves of the trajectories, as well as the statistics of the fit parameters [14, 22] in each step (Fig. 2c, d; Additional file 1: Fig. S1), QDs-RBV experienced a slow and anomalous diffusion movement with $D$ of $0.0030 \pm 0.0008 \mu \mathrm{m}^{2} / \mathrm{s}$ and $\alpha$ of $0.71 \pm 0.09$ in step 1 (black), travelled fast and directly with $D$ of $0.011 \pm 0.002 \mu \mathrm{m}^{2} / \mathrm{s}$ and $V$ of $0.072 \pm 0.010 \mu \mathrm{m} / \mathrm{s}$ in step 2 (red), then moved with a slow and anomalous diffusion motion mode again with $D$ of $0.0021 \pm 0.0004 \mathrm{\mu m}^{2} / \mathrm{s}$ and $\alpha$ of $0.52 \pm 0.08$ in step 3 (blue). The directed motion segments (step 2) started at $60 \pm 6 \mathrm{~s}$ and ended at $100 \pm 4 \mathrm{~s}$. Thus, the endocytosis mechanism of baculovirus was detailedly dissected and confirmed. Moreover, the vesicle-vesicle fusion hypothesis based on conventional transmission electron microscopy (TEM) technique [8] was verified by the observed three steps of endocytosis.

\section{Dynamic interaction between QDs-RBVs and acidic endosomes}

Live Sf9 cells labeled by LysoTracker Green (an acidic endosome dye) were infected with QDs-RBVs for SVT. Figure 3a showed that most signals of QDs-RBVs overlapped with those of acidic endosomes, indicating that baculovirus entered acidic endosomes. Typical timelapse images showed single QDs-RBV moving to and colliding with an acidic endosome (Fig. 3b; Additional file 1: Fig. S2a). By analyzing 30 valid trajectories, it was found that QDs-RBV travelled fast and directly to acidic endosome for $36 \pm 4$ s with $D$ of $0.0083 \pm 0.0008 \mu \mathrm{m}^{2} / \mathrm{s}$ and $V$ of $0.062 \pm 0.009 \mu \mathrm{m} / \mathrm{s}$ (red), and then collided with acidic endosome in a slow and anomalous diffusion manner 


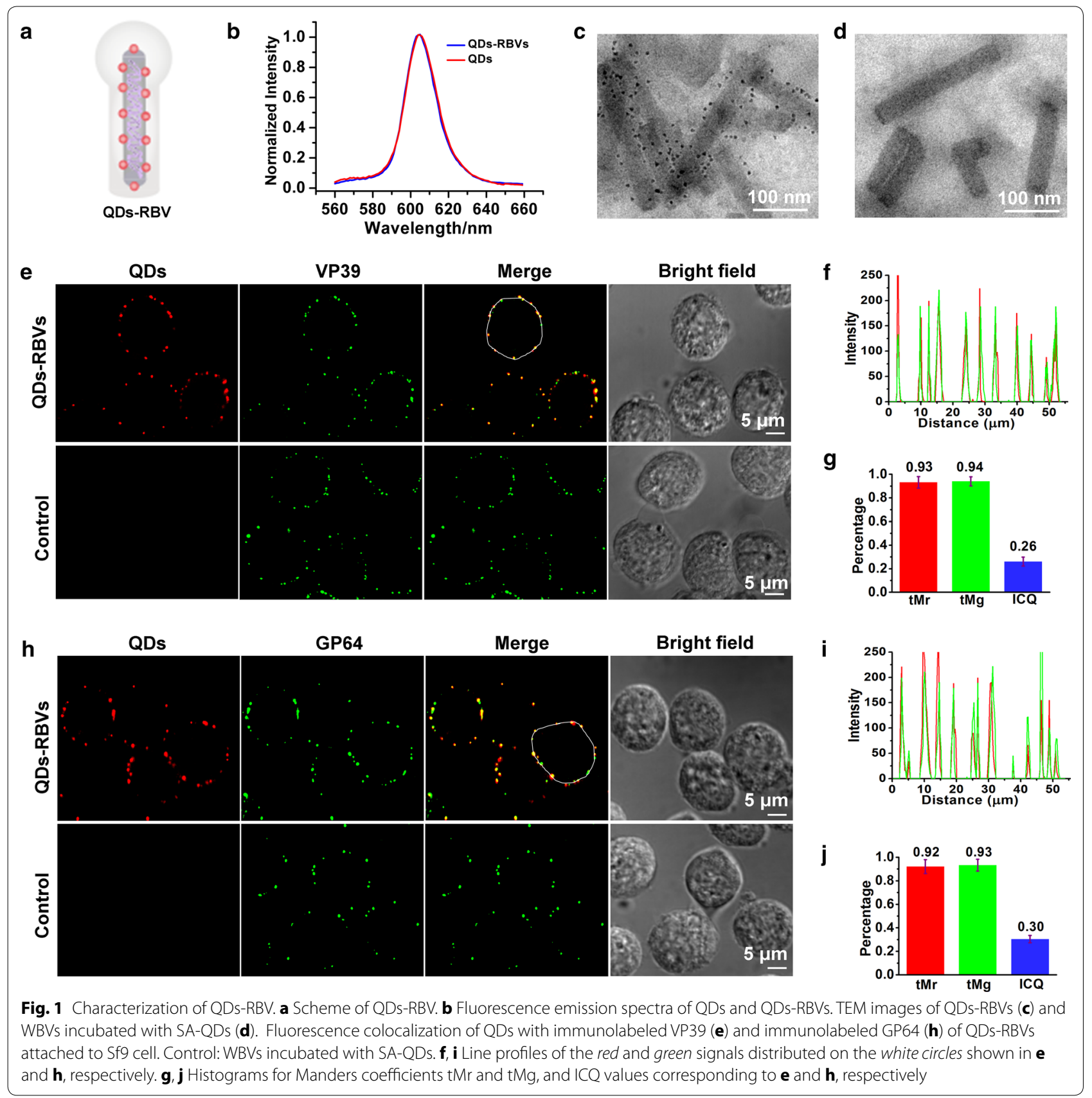

for $36 \pm 6 \mathrm{~s}$ with $D$ of $0.0013 \pm 0.0003 \mu \mathrm{m}^{2} / \mathrm{s}$ and $\alpha$ of $0.62 \pm 0.07$ (blue) (Fig. 3c, d; Additional file 1: Fig. S2b$\mathrm{S} 2 \mathrm{~g})$. Thus direct evidence for baculovirus fusion with acidic endosome after the endocytosis [24] was provided.

Live Sf9 cells treated or untreated with Bafilomycin A1 (a drug prevents the formation of acidic endosomes) were infected by QDs-RBVs. As shown in Fig. 3e, $\mathrm{f}$ and Additional file 1: Fig. S2h-S2m, compared with the fast and directed motion mode of QDs-RBV with $D$ of $0.014 \pm 0.004 \mu \mathrm{m}^{2} / \mathrm{s}$ and $V$ of $0.11 \pm 0.03 \mu \mathrm{m} / \mathrm{s}(\mathrm{n}=30)$ in the untreated cells (red), QDs-RBV in the treated cells experienced a slow and anomalous diffusion movement with $D$ of $0.0024 \pm 0.0003 \mu \mathrm{m}^{2} / \mathrm{s}$ and $\alpha$ of $0.51 \pm 0.08$ $(\mathrm{n}=30)$ (blue). Moreover, the yield of baculoviruses propagated in the treated cells significantly decreased compared to that in the control (Fig. 3g). Thus combining SVT and drug inhibition assays, it is proved that the formation of acidic endosomes and their fusion with baculovirus were the prerequisites for baculovirus retrograde transportation. 


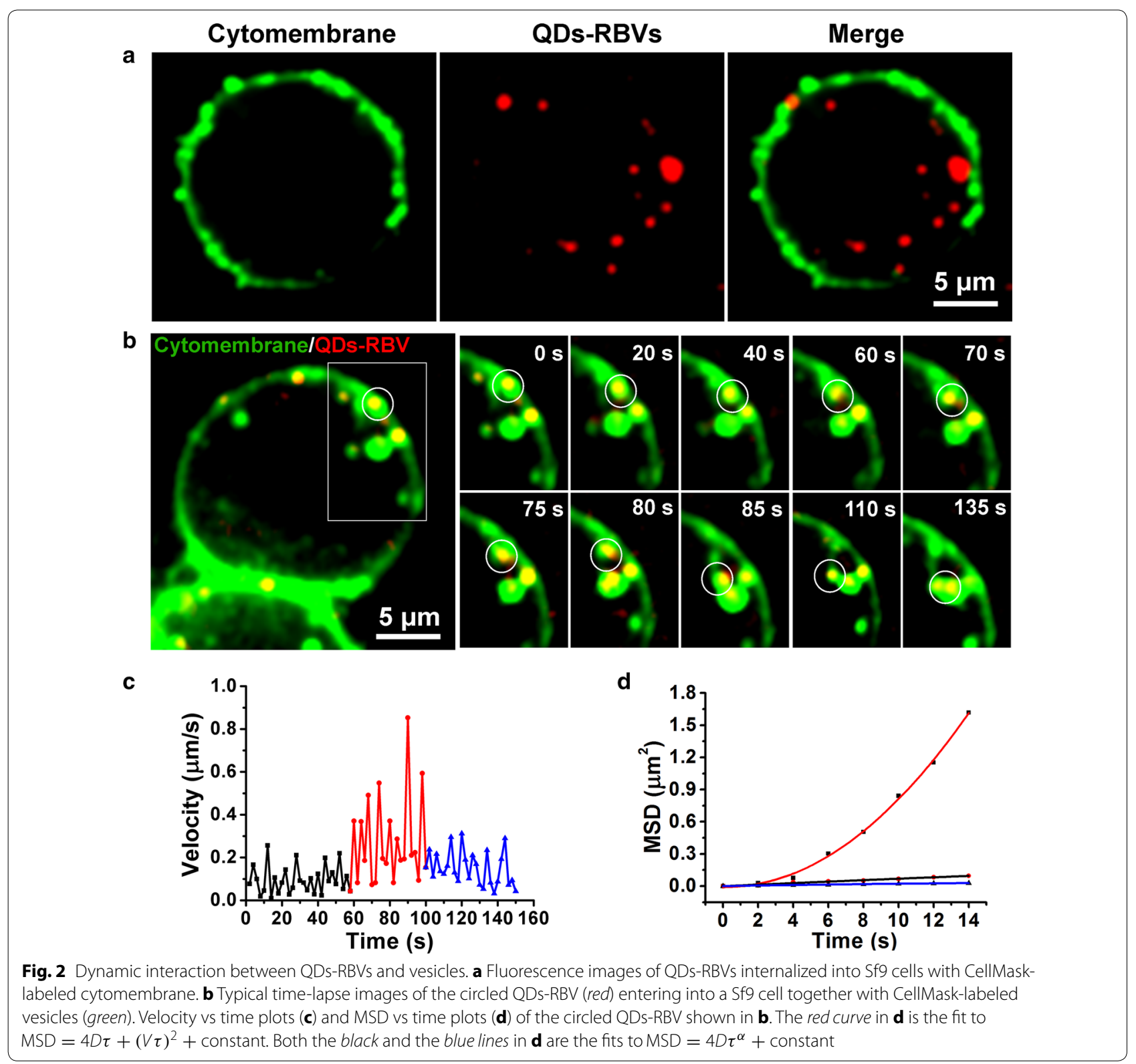

\section{Interaction between QDs-RBVs and actins}

Sf9 cells infected by QDs-RBVs were fixed and permeabilized before incubating with cell-impermeable actin dye Phalloidin-FITC owing to the lack of cell-permeable dyes for labeling actins in live cells. Remarkably, most signals of QDs-RBVs were closely adjacent to those of actins (Fig. 4a; Additional file 1: Fig. S3a), which illustrated baculoviruses being trailed by actin tails during intracellular movement. According to previous virology studies [25, 26], after the fusion with acidic endosomes, baculoviruses could induce the disruption and rearrangement of the continuous filamentous actins to form tail-like actin structures, which subsequently drive baculovirus to the perinuclear region. Thus the postfusion infection event was verified at the single-virus level.

Live Sf9 cells treated or untreated with Cytochalasin D (an actin-polymerization inhibitor) were also infected with QDs-RBVs. As shown in Fig. 4b, c and Additional file 1: Fig. S3b-S3g, QDs-RBV moved rapidly and directly with $D$ of $0.015 \pm 0.004 \mu \mathrm{m}^{2} / \mathrm{s}$ and $V$ of $0.12 \pm 0.03 \mu \mathrm{m} / \mathrm{s}$ $(\mathrm{n}=30)$ in the untreated cells (red), whereas QDs-RBV experienced a slow and anomalous diffusion movement with $D$ of $0.0023 \pm 0.0005 \mu^{2} / \mathrm{s}$ and $\alpha$ of $0.63 \pm 0.08$ $(\mathrm{n}=30)$ in the treated cells (blue). Moreover, Fig. $4 \mathrm{~d}$ showed that the yield of baculoviruses propagated in Nocodazole (a microtubule-depolymerization drug) 


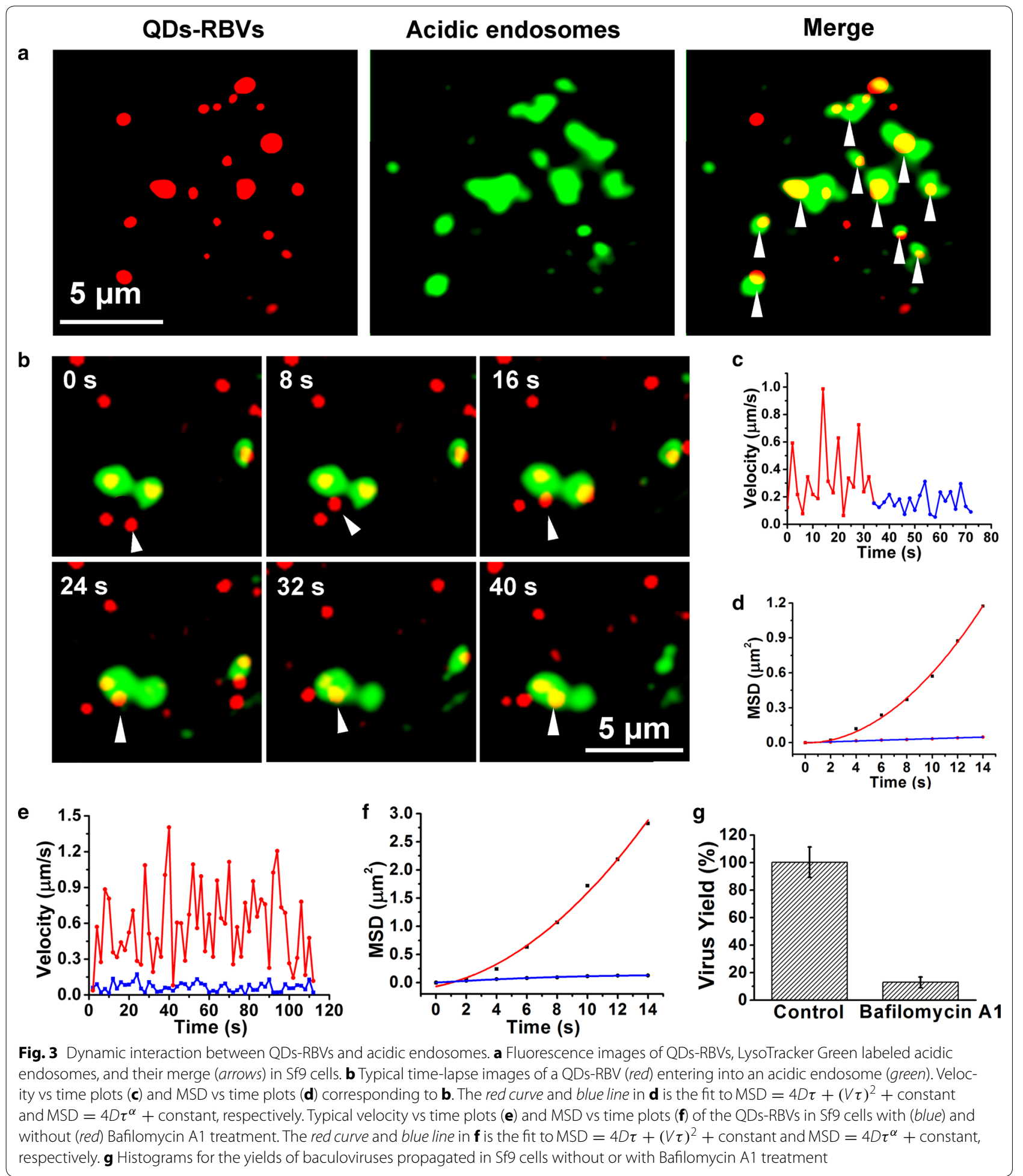

treated cells was as high as that in the control. In contrast, disturbing actin polymerization significantly influenced baculovirus propagation. All these results confirmed that, although most viruses take microtubules as their highways for transportation [9, 27], baculovirus trafficking towards perinuclear region depends on actin. 

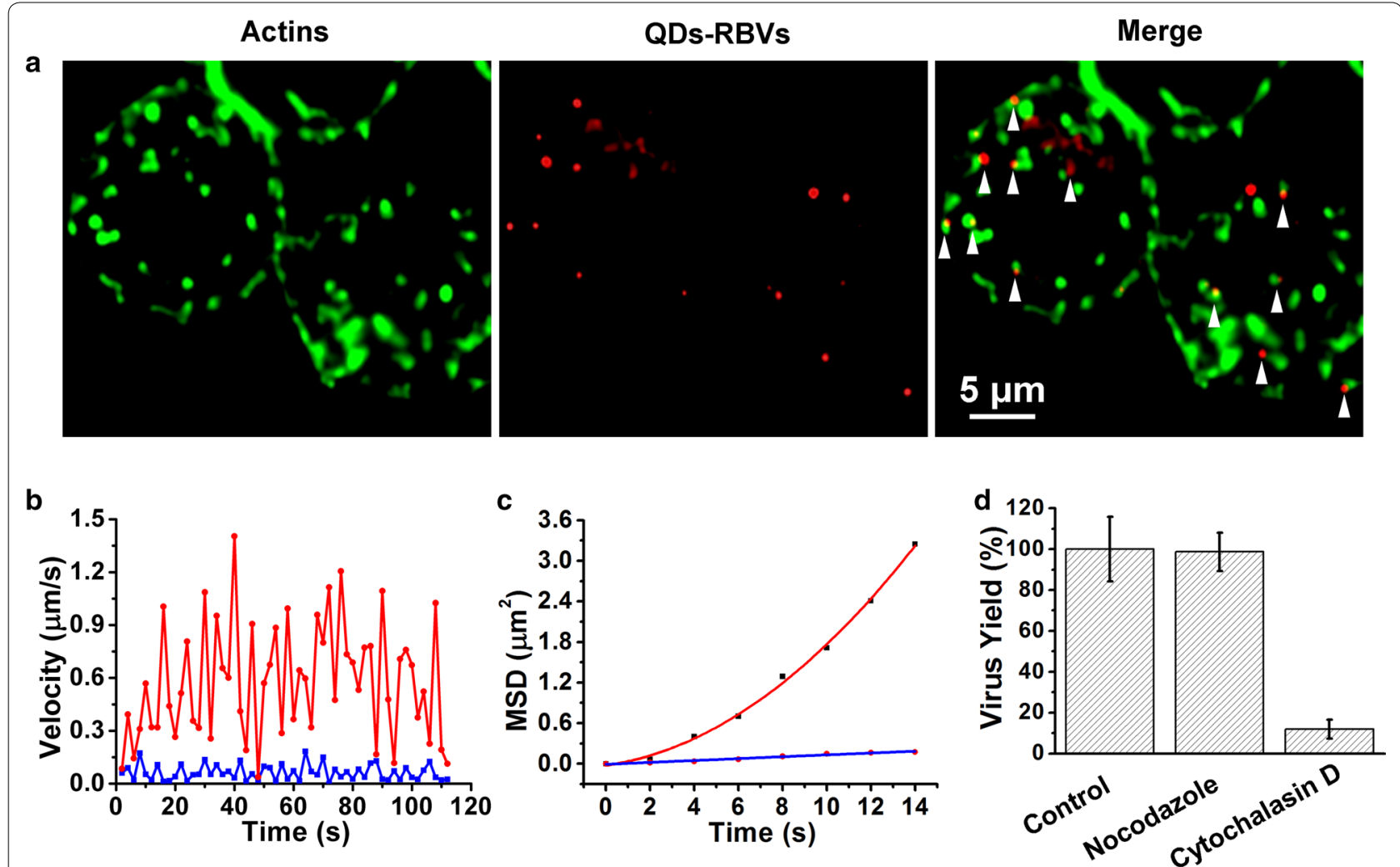

Fig. 4 Interaction between QDs-RBVs and actins. a Fluorescence images of QDs-RBVs, phalloidin-FITC labeled actins and their merge (arrows) in Sf9 cells. Typical velocity vs time plots (b) and MSD vs time plots (c) of the QDs-RBVs in Sf9 cells with (blue) and without (red) cytochalasin D treatment. The red curve and blue line in $\mathbf{c}$ is the fit to MSD $=4 D \tau+(V \tau)^{2}+$ constant and MSD $=4 D \tau^{\alpha}+$ constant, respectively. $\mathbf{d}$ Histograms for the yields of baculoviruses propagated in untreated, nocodazole-treated and cytochalasin D-treated Sf9 cells

\section{Interaction between QDs-RBVs and nucleus}

The nuclei of live Sf9 cells were labeled with Hoechst 33342 for imaging. No obvious cytotoxicity was observed by the incubation of Hoechst 33342 with different incubation concentration (Additional file 1: Fig. S4a) or different incubation time (Additional file 1: Fig. S4b). Abundant immunofluorescence of NPC was observed around nucleus (Fig. 5a), implying that nuclear pores vastly distributed on nuclear membranes and might play an essential role in baculovirus nuclear import. Moreover, while some QDs-RBVs were in the nucleus (white arrows), other QDs-RBVs colocalized with NPC (green arrows), suggesting that baculoviruses entered into nucleus through their interactions with nuclear pores. This is in accordance with previous TEM images of baculoviruses docking on nuclear pores [28]. Subsequently, Sf9 cells were microinjected with FITCWGA. Results showed that the distribution of FITCWGA around nucleus (Fig. 5b) was similar to that of
NPC (Fig. 5a), indicating that WGA bound to nuclear pores and thus blocked the access to nucleus [29]. Fig. 5b also showed that no QDs-RBV was internalized into the nucleus of WGA-microinjected cell. On the contrary, without WGA microinjection, QDs-RBVs entered into the nucleus (the control), further proving that baculoviruses take nuclear pores as the channels for nuclear import [30].

Previously, we have found that baculovirus moved directly and slowly in the nucleus after nuclear entry [11]. However, destination of the intranuclear baculoviruses remains unclear. Notably, most intranuclear QDsRBVs were found in the non-nucleic acids area (Fig. 5c). It was found that QDs-RBVs in this area moved with an anomalous diffusion and slow motion mode with $D$ of $0.0022 \pm 0.0006 \mu \mathrm{m}^{2} / \mathrm{s}$ and $\alpha$ of $0.81 \pm 0.04(\mathrm{n}=30)$ (Fig. 5d, e; Additional file 1: Fig. S4c-S4f), indicating that this region might be the terminal of baculovirus retrograde transportation. 

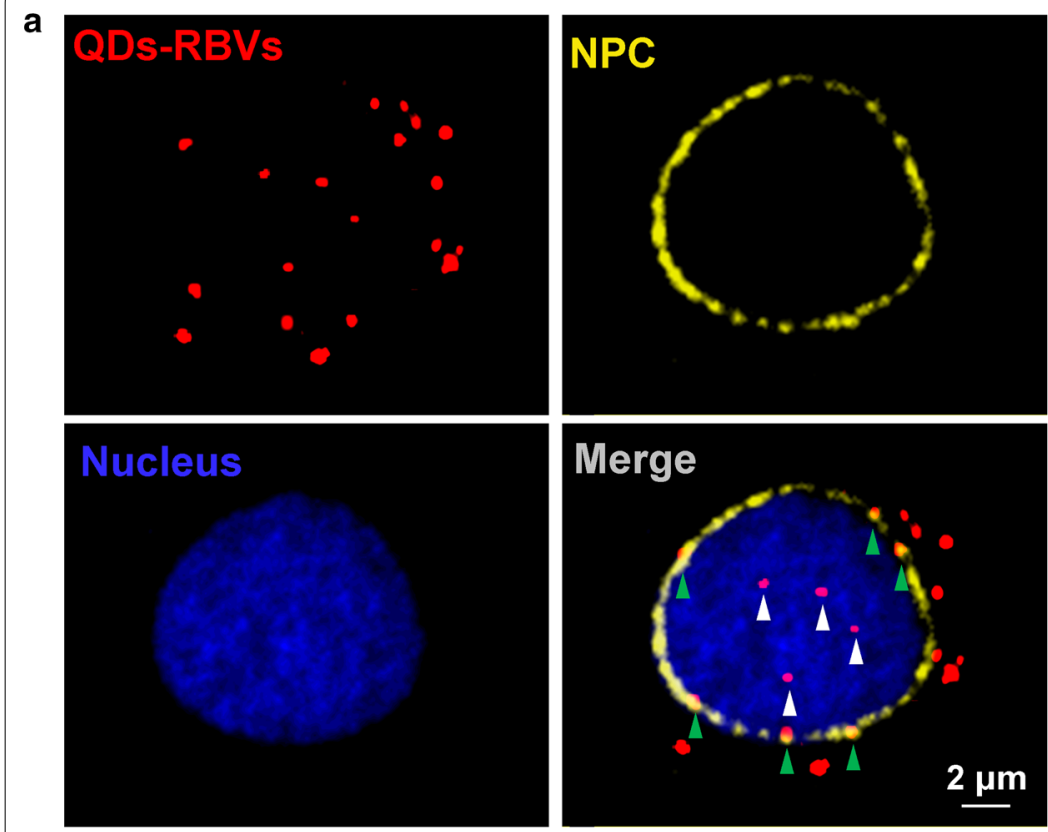

C

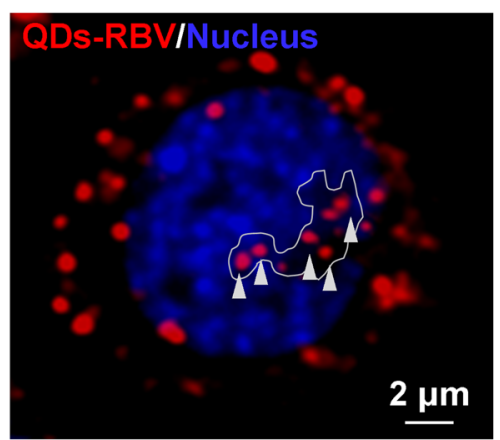

d

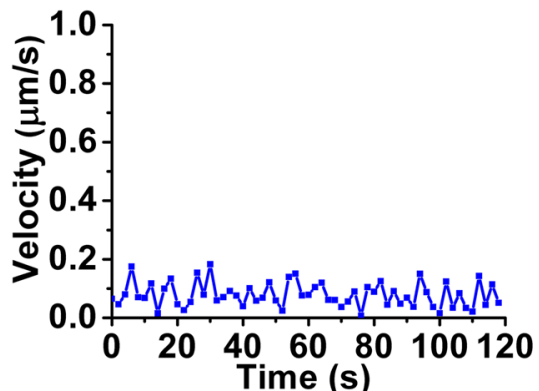

b
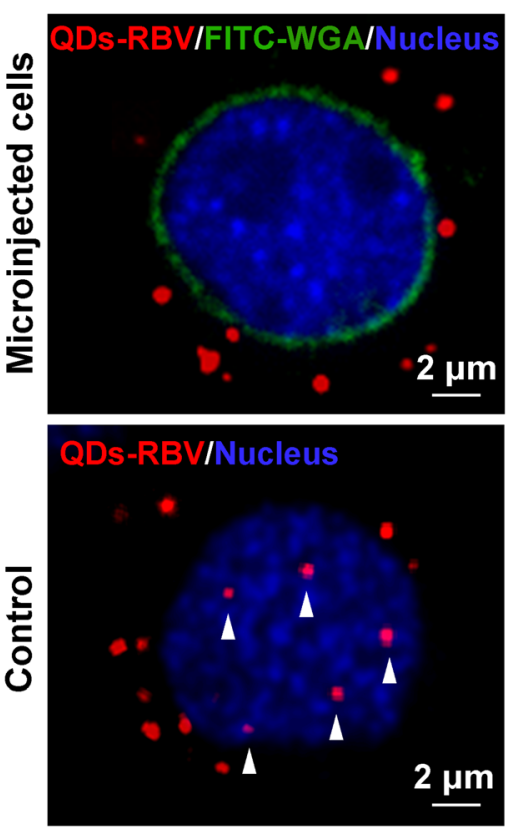

e

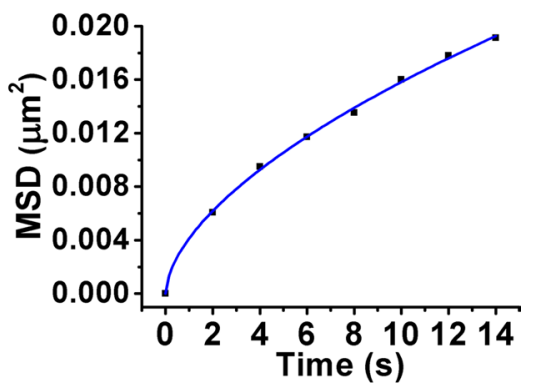

Fig. 5 Interaction between QDs-RBVs and nucleus. a Fluorescence images of QDs-RBVs, immunolabeled NPC, Hoechst 33342-labeled nucleus and their merge in Sf9 cells. b Fluorescence images of QDs-RBVs in Sf9 cell microinjected with or without (the control) FITC-WGA. c Fluorescence images of QDs-RBVs confined in the non-nucleic acids area (white-line defined) of nucleus. Typical velocity vs time plots (d) and MSD vs time plots (e) of the arrowed QDs-RBVs shown in $\mathbf{c}$. The curve in $\mathbf{e}$ is the fit to MSD $=4 D \tau^{\alpha}+$ constant

\section{Comprehensive retrograde transportation model of baculovirus}

Thus the comprehensive retrograde trafficking of baculovirus is dissected at the single-virus level based on the QD-labeling of baculovirus internal component and the SVT (Fig. 6): virus being endocytosed into cell by vesicle trapping and vesicle-vesicle fusion, moving towards and fusing with acidic endosome for the release in cytoplasm, being driven by actin tails towards nuclear periphery, translocating into nucleus through nuclear pore, and arriving at the destination of retrograde transportation finally.

\section{Conclusions}

By employing SVT and QD-labeling of virus internal component, combined with drug inhibition assays, retrograde transportation of individual baculovirus in host cells was dynamically tracked in real time. By analyzing the behaviors of individual baculovirus interacting with key related cellular structures including vesicles, acidic endosomes, actins, nuclear pores and nuclei, critical infection events both before and after the fusion were dissected. These results provide detailed scenarios and dynamic insights into baculovirus infection. More importantly, this is the first time that the whole retrograde 


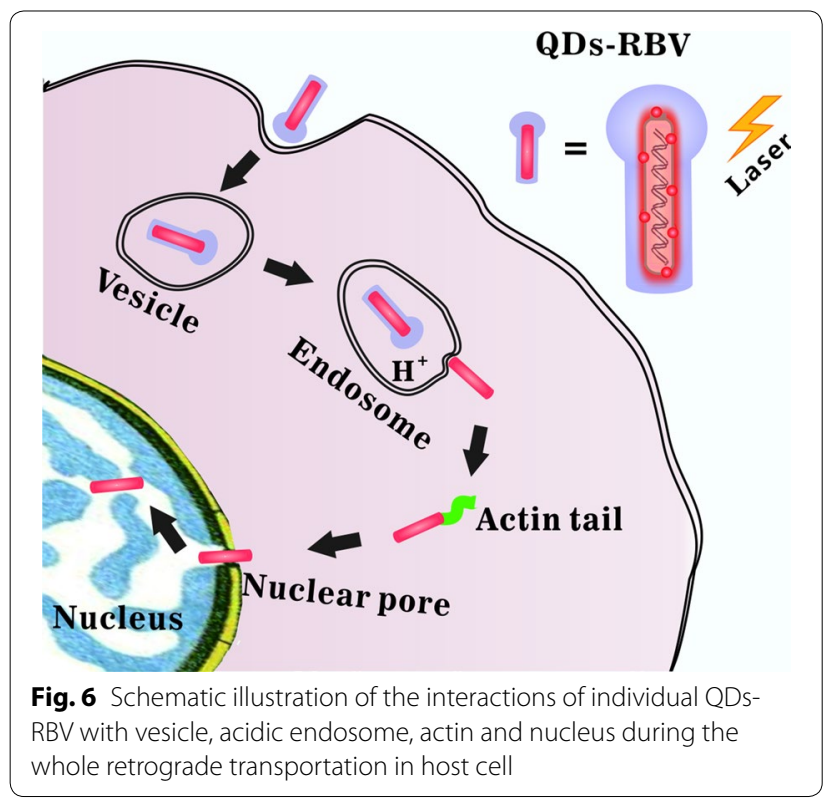

transportation of baculovirus is visualized at the singlevirus level. Thus deeper understanding of baculovirus trafficking and virus-cell interaction was enabled.

\section{Additional file}

Additional file 1. Statistics of the dynamic interactions between QDsRBVs and cellular structures.

\section{Abbreviations}

QDs: quantum dots; SVT: single virus tracking; RBV: recombinant baculovirus; Sf9: Spodoptera frugiperda 9; MOI: multiplicity of infection; WBV: wild type baculovirus; TEM: transmission electron microscope; NPC: nuclear pore complexes; FITC: fluorescein isothiocyanata; WGA: wheat germ agglutinin; MTT: [3-(4,5-dimethylthiazol-2-yl)-2,5-diphenyltetrazolium bromide]; TCID50: 50\% tissue culture infective dose; ICQ: intensity correlation quotient; MSD: mean square displacement.

\section{Authors' contributions}

$L W$ and $Y L$ conceived and designed the experiments, and wrote the manuscript. LW, ZHZ and AAL performed the experiments. LC and LJZ analyzed the data. JA prepared the materials ZLZ and HZW contributed to the data interpretation. YL and DWP coordinated and supervised the work. All authors read and approved the final manuscript.

\section{Author details}

${ }^{1}$ Key Laboratory of Analytical Chemistry for Biology and Medicine (Ministry of Education), College of Chemistry and Molecular Sciences, State Key Laboratory of Virology, The Institute for Advanced Studies, and Wuhan Institute of Biotechnology, Wuhan University, Wuhan 430072, People's Republic of China. ${ }^{2}$ State Key Laboratory of Virology, Wuhan Institute of Virology, Chinese Academy of Sciences, Wuhan 430071, People's Republic of China.

\section{Acknowledgements}

We thank Prof. Wei-Hua Huang and Xin-Wei Zhang for their help in microinjection experiments.

\section{Competing interests}

The authors declare that they have no competing interests.

\section{Availability of data and materials}

The datasets supporting the conclusions of this article are included within the article and its Additional file 1.

\section{Consent for publication}

The authors declare that they are consent for the publication of this manuscript.

\section{Ethics approval and consent to participate}

The authors declare that they are consent to participate this work.

\section{Funding}

This work was supported by the National Natural Science Foundation of China (Nos. 21275111, 21535005), the National High Technology Research and Development Program (863 Program, No. 2013AA032204), the National Basic Research Program of China (973 Program, No. 2011CB933600), the 111 Project (No. 111-2-10), the China Scholarship Council (No. 201406275115), and Collaborative Innovation Center for Chemistry and Molecular Medicine.

\section{Publisher's Note}

Springer Nature remains neutral with regard to jurisdictional claims in published maps and institutional affiliations.

Received: 18 November 2016 Accepted: 26 April 2017 Published online: 06 May 2017

\section{References}

1. Kaye D, Pringle CR. Avian influenza viruses and their implication for human health. Clin Infect Dis. 2005;40:108-12.

2. Brandenburg B, Zhuang XW. Virus trafficking-learning from single-virus tracking. Nat Rev Microbiol. 2007;5:197-208.

3. Lara HH, Ixtepan-Turrent L, Garza-Treviño EN, Rodriguez-Padilla C. PVPcoated silver nanoparticles block the transmission of cell-free and cellassociated HIV-1 in human cervical culture. J Nanobiotechnol. 2010;8:15.

4. Wen L, Lin Y, Zhang ZL, Lu W, Lv C, Chen ZL, Wang HZ, Pang DW. Intracellular self-assembly based multi-labeling of key viral components: envelope, capsid and nucleic acids. Biomaterials. 2016;99:24-33.

5. Liu SL, Wang ZG, Zhang ZL, Pang DW. Tracking single viruses infecting their host cells using quantum dots. Chem Soc Rev. 2016;45:1211-24.

6. Chan WCW, Nie SM. Quantum dot bioconjugates for ultrasensitive nonisotopic detection. Science. 1998;281:2016-8.

7. Joo Kl, Lei YN, Lee CL, Lo J, Xie JS, Hamm-Alvarez SF, Wang P. Site-specific labeling of enveloped viruses with quantum dots for single virus tracking. ACS Nano. 2008;2:1553-62.

8. Liu HB, Liu Y, Liu SL, Pang DW, Xiao GF. Clathrin-mediated endocytosis in living host cells visualized through quantum dot labeling of infectious hematopoietic necrosis virus. J Virol. 2011;85:6252-62.

9. Liu SL, Zhang LJ, Wang ZG, Zhang ZL, Wu QM, Sun EZ, Shi YB, Pang DW. Globally visualizing the microtubule-dependent transport behaviors of influenza virus in live cells. Anal Chem. 2014;86:3902-8.

10. Zhang Y, Ke XL, Zheng ZH, Zhang CL, Zhang ZF, Zhang FX, Hu QX, He ZK, Wang HZ. Encapsulating quantum dots into enveloped virus in living cells for tracking virus infection. ACS Nano. 2013;7:3896-904.

11. Wen $L$, Lin $Y$, Zheng $Z H$, Zhang ZL, Zhang LJ, Wang LY, Wang HZ, Pang DW. Labeling the nucleocapsid of enveloped baculovirus with quantum dots for single-virus tracking. Biomaterials. 2014;35:2295-301.

12. Monteiro F, Carinhas N, Carrondo MJT, Bernal V, Alves PM. Toward systemlevel understanding of baculovirus-host cell interactions: from molecular fundamental studies to large-scale proteomics approaches. Front Microbiol. 2012;3:391.

13. Chou TC, Hsu W, Wang CH, Chen YJ, Fang JM. Rapid and specific influenza virus detection by functionalized magnetic nanoparticles and mass spectrometry. J Nanobiotech. 2011;9:52.

14. Liu AA, Zhang ZF, Sun EZ, Zheng ZH, Zhang ZL, Hu QX, Wang HZ, Pang DW. Simultaneous visualization of parental and progeny viruses by a capsid-specific HaloTag labeling strategy. ACS Nano. 2016;10:1147-55. 
15. Manzo C, Garcia-Parajo MF. A review of progress in single particle tracking: from methods to biophysical insights. Rep Prog Phys. 2015;78:124601.

16. Liu SL, Zhang ZL, Tian ZQ, Zhao HS, Liu HB, Su EZ, Xiao GF, Zhang WP, Wang $\mathrm{HZ}$, Pang DW. Effectively and efficiently dissecting the infection of influenza virus by quantum-dot-based single-particle tracking. ACS Nano. 2012:6:141-50

17. Ruthardt N, Lamb DC, Bräuchle C. Single-particle tracking as a quantitative microscopy-based approach to unravel cell entry mechanisms of viruses and pharmaceutical nanoparticles. Mol Ther. 2011;19:1199-211.

18. Summers MD, Volkman L. Comparison of biophysical and morphological properties of occluded and extracellular nonoccluded baculovirus from in vivo and in vitro host systems. J Virol. 1976;17:962-72.

19. Deng F, Wang R, Fang MG, Jiang Y, Xu XS, Wang HZ, Chen XW, Arif BM, Guo L, Wang HL, Hu ZH. Proteomics analysis of Helicoverpa armigera single nucleocapsid nucleopolyhedrovirus identified two new occlusion-derived virus-associated proteins, HA44 and HA100. J Virol. 2010;81:9377-85.

20. Volkman LE, Goldsmith PA. Mechanism of neutralization of budded Autographa californica nuclear polyhedrosis virus by a monoclonal antibody: inhibition of entry by adsorptive endocytosis. J Virol. 1985:143:185-95.

21. Long G, Pan XY, Kormelink R, Vlak JM. Functional entry of baculovirus into insect and mammalian cells is dependent on clathrin-mediated endocytosis. J Virol. 2006;80:8830-3.
22. Bruin KD, Ruthardt N, Gersdorff KV, Bausinger R, Wagner E, Ogris M, Bräuchle C. Cellular dynamics of EGF receptor-targeted synthetic viruses. Mol Ther. 2007:15:1297-305.

23. Rust MJ, Lakadamyali M, Zhang F, Zhuang XW. Assembly of endocytic machinery around individual influenza viruses during viral entry. Nat Struct Mol Biol. 2004;11:567-73

24. Blissard GW, Wenz JR. Baculovirus gp64 envelope glycoprotein is sufficient to mediate $\mathrm{pH}$-dependent membrane fusion. J Virol. 1992;66:6829-35.

25. Ohkawa T, Volkman LE, Welch MD. Actin-based motility drives baculovirus transit to the nucleus and cell surface. J Cell Biol. 2010;190:187-95.

26. Marek M, Merten OW, Francis-Devaraj F, Oers MM. Essential C-terminal region of the baculovirus minor capsid protein VP80 binds DNA. J Virol. 2012;86:1728-38

27. Greber UF, Way M. A superhighway to virus infection. Cell. 2006:124:741-54

28. Au S, Panté N. Nuclear transport of baculovirus: revealing the nuclear pore complex passage. J Struct Biol. 2012;177:90-8.

29. Kylberg K, Björk P, Fomproix N, Ivarsson B, Wieslander L, Daneholt B. Exclusion of mRNPs and ribosomal particles from a thin zone beneath the nuclear envelope revealed upon inhibition of transport. Exp Cell Res. 2010;316:1028-38.

30. Au S, Wu W, Panté N. Baculovirus nuclear import: open, nuclear pore complex (NPC) sesame. Viruses. 2013;5:1885-900.

\section{Submit your next manuscript to BioMed Central and we will help you at every step:}

- We accept pre-submission inquiries

- Our selector tool helps you to find the most relevant journal

- We provide round the clock customer support

- Convenient online submission

- Thorough peer review

- Inclusion in PubMed and all major indexing services

- Maximum visibility for your research

Submit your manuscript at www.biomedcentral.com/submit 